\title{
CFD Modelling of the Effect of Oscillating Jets on Streaming Heat Losses in a Torus-shaped Thermoacoustic Engine
}

\author{
J.A. Lycklama à Nijeholt ${ }^{1 *}$, M.E.H. Tijani ${ }^{1}$, N.B. Siccama ${ }^{2}$ \\ ${ }^{1}$ Heat \& Biorefinery Technology, Biomass \& Energy Efficiency, ECN, P.O. Box 1, 1755 LE, Petten, The Netherlands. \\ *Corresponding author's e-mail: lycklama@ecn.nl \\ 2 Safety and waste, ResearchE Innovation, NRG, The Netherlands.
}

Keywords: Thermoacoustics, CFD, Acoustic Streaming, Oscillating Heat Transfer, Heat Engine

\section{Introduction}

A thermosacoustic engine converts heat into acoustic energy. A high temperature engine utilizes heat from a burner to drive the engine. Due to the high driving temperature acoustic streaming can be a strong heat loss mechanism that should be limited as much as possible. Linear acoustic simulation tools cannot be used to predict streaming nor its resulting heat loss. Therefore, computational fluid dynamics (CFD) has been used to simulate the streaming in a torus-shaped thermoacoustic engine. The present study builds upon previous CFD modeling and experimental studies from ECN on the high temperature TA engine and presented in [1] and [2]. Aim of the present work is to investigate the effect of oscillating helium jets originating from the heat exchangers on the degree of the streaming heat losses in the thermal buffer tube (TBT).

\section{CFD model}

The CFD model simulates the engine which consists of a torus-shaped part with heat exchangers and regenerator. The engine is connected to the resonator with an acoustic load. The resonator has also been included in the CFD geometry. The experimental geometry has been simplified into a two dimensional model in order to save CPU time. The streaming velocity field in the torus has been calculated by means of time-averaging of the oscillating flow. The convective streaming heat loss in the TBT has been calculated from the predicted thermal load of the TBT heat exchanger. The helium slots in the heat exchangers have been included in the CFD model in order to investigate the effect of the oscillating jets on the streaming flow pattern and streaming heat loss in the TBT.

\section{Results}

Jets from the heat exchangers enhance thermal mixing because of the additional production of turbulence. The CFD study shows however, a previously unknown and much stronger adverse effect of the helium jets. The helium jets enhance the convective streaming vortex present in the TBT resulting in much higher streaming velocities. Consequently, the convective heat loss in the TBT is also strongly increased. The physical flow mechanism for enhancement of the streaming flow rate by means of the jets is not yet fully understood.

The calculated temperature profile in the TBT and the thermal load of the TBT heat exchanger have been compared to the experimental data. The CFD results showed similar temperature profiles in the TBT and similar trends in the convective streaming heat loss. This 
qualitative good agreement showed that the two-dimensional model approximation could be applied to demonstrate the effect of jets.

\section{References}

[1] Lycklama a Nijeholt, J.A.; Tijani, M.E.H.; Spoelstra, S.; Loginov, M.S.; Kuczaj, A.K.; "CFD modeling of streaming phenomena in a torus-shaped thermoacoustic engine ", 19th International Congress on Sound and Vibration (ICSV19), Vilinius, Litouwen, July 8-12, 2012.

[2] Tijani,M.E.H.; Spoelstra,S.; "A hot air driven thermoacoustic-Stirling engine”, Applied Thermal Engineering (Elsevier), 2013, Ed.61, p.866-870. 